This item was submitted to Loughborough's Research Repository by the author.

Items in Figshare are protected by copyright, with all rights reserved, unless otherwise indicated.

\title{
Acoustic propagation in dispersions in the long wavelength limit
}

PLEASE CITE THE PUBLISHED VERSION

http://dx.doi.org/10.1137/04061698X

PUBLISHER

(c) Society for Industrial and Applied Mathematics

VERSION

VoR (Version of Record)

LICENCE

CC BY-NC-ND 4.0

REPOSITORY RECORD

Pinfield, Valerie J., O.G. Harlen, Malcolm J.W. Povey, and B.D. Sleeman. 2012. "Acoustic Propagation in Dispersions in the Long Wavelength Limit". figshare. https://hdl.handle.net/2134/11081. 
This item was submitted to Loughborough's Institutional Repository (https://dspace.lboro.ac.uk/) by the author and is made available under the following Creative Commons Licence conditions.

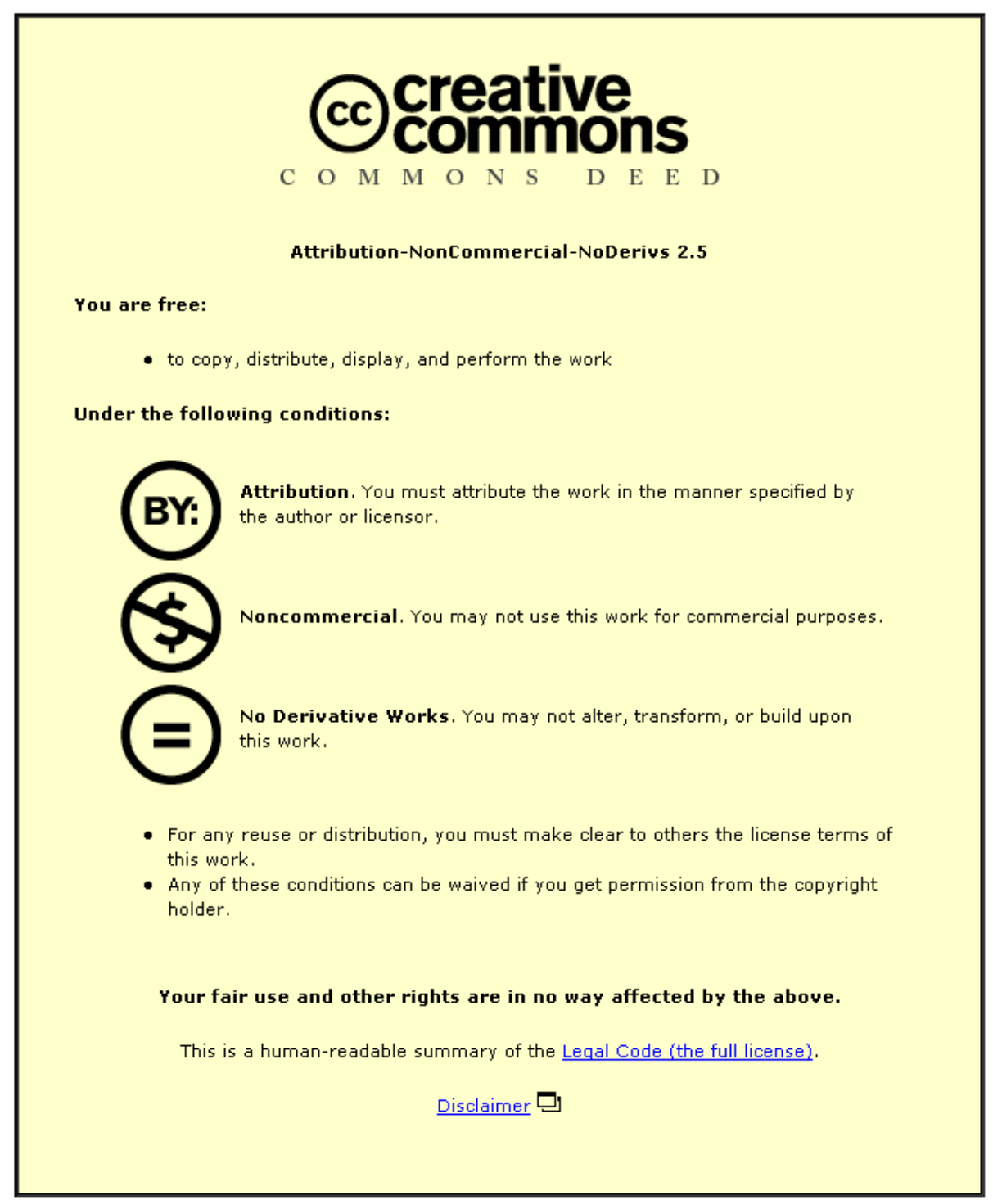

For the full text of this licence, please go to: http://creativecommons.org/licenses/by-nc-nd/2.5/ 


\title{
ACOUSTIC PROPAGATION IN DISPERSIONS IN THE LONG WAVELENGTH LIMIT*
}

\author{
V. J. PINFIELD ${ }^{\dagger}$, O. G. HARLEN ${ }^{\ddagger}$, M. J. W. POVEY ${ }^{\dagger}$, AND B. D. SLEEMAN ${ }^{\ddagger}$
}

\begin{abstract}
The problem of scattering of ultrasound by particles in the long wavelength limit has a well-established solution in terms of Rayleigh expansions of the scattered fields. However, this solution is ill-conditioned numerically, and recent work has attempted to identify an alternative method. The scattered fields have been expressed as a perturbation expansion in the parameter $K a$ (the wavenumber multiplied by the particle radius), which is small in the long wavelength region. In the work reported here the problem has been formulated so as to be valid for all values of the thermal wavelength, which varies in order of magnitude, from much smaller to much larger than the particle size in the long wavelength region. Thus the present solution overlaps the limiting solutions for very small thermal wavelength (geometric theory) and very large thermal wavelength (low frequency) previously reported. Close agreement is demonstrated with the established Rayleigh expansion solution.
\end{abstract}

Key words. Helmholtz equation, scattering theory, ultrasound spectroscopy, dispersions

AMS subject classifications. 35C10, 35J05, 35P25, 76Q05

DOI. $10.1137 / 04061698 \mathrm{X}$

1. Introduction. Ultrasound spectroscopy is an increasingly popular technique for characterizing the physical properties of dispersions, emulsions, gels, and solutions of biomolecules. It is a noninvasive technique that can address the extensive range of particle sizes encountered in many particulate systems and can be used with optically opaque materials. The technique has been adopted in manufacturing processes in the food and chemical industries. Ultrasonic instruments may be used to determine particle size distribution and/or concentration of the dispersed particles. In order to do this, it is necessary to use a strong theoretical basis to relate the ultrasound properties, i.e., velocity and attenuation, to the particle size and physical properties of the materials.

The problem of scattering of sound waves by a single spherical object (a fluid droplet) was solved by Rayleigh [1] and later refined by Epstein and Carhart [2]. A similar problem, with solid particles, was addressed by Allegra and Hawley [3]. Their solution is referred to as ECAH. The scattered fields are expanded as spherical harmonics in order to allow the application of boundary conditions at the particle surface. Although the solution is analytically exact, its numerical solution can be troublesome, because the matrix equation is ill-conditioned, and the series does not converge uniformly. In addition, calculation of spherical Bessel functions at large complex arguments is imprecise, and at large distances the Hankel functions oscillate rapidly. Such numerical limitations cause difficulty in applying the ultrasound method in practical applications.

* Received by the editors October 14, 2004; accepted for publication (in revised form) April 27, 2005; published electronically December 30, 2005. This research was supported by the UK Engineering and Physical Research Council (EPSRC), grant GR/L/51034.

http://www.siam.org/journals/siap/66-2/61698.html

${ }^{\dagger}$ Procter Department of Food Science, University of Leeds, Leeds LS2 9JT, UK (v.j.pinfield@leeds. ac.uk, m.j.w.povey@leeds.ac.uk).

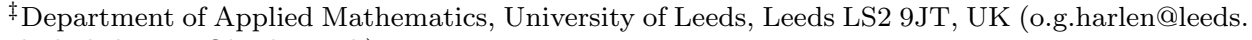
ac.uk, b.d.sleeman@leeds.ac.uk). 
The aim of the present work in this area has been to formulate a numerically stable solution to the single scatterer problem in such a way as to allow its potential extension to multiple scatterers and to nonspherical scatterers. The frequency range of interest is termed the long wavelength limit, in which the wavelength of the propagating acoustic wave is much larger than the size of the droplet $(K a \ll 1$, where $K$ is the wavenumber and $a$ the radius of the particles). In this case, Kleinman's approach can be applied, in which the problem is reformulated to satisfy the radiation condition and uses a perturbation series solution in powers of Ka. At the lowest frequencies, at which the wavelength of the thermal waves produced by scattering is also much larger than the particle radius ( $L a \ll 1$, where $L$ is the thermal wavenumber), the same technique can be applied to the thermal wave. The method was applied to single particle scattering in the low-frequency potential scattering theory (LFPST) previously published (Harlen et al. [4]). A later paper (Harlen et al. [5]) considered the case $L a \gg 1$, where the thermal wavelength is much smaller than the particle size, and the Kleinman series expansion in $L a$ cannot be used. In this case, a geometric theory method was developed to approximate the solution for the thermal waves while retaining the Kleinman technique for the propagational waves.

In the work reported here, the Kleinman principles have again been used to separate the radiative terms of the waves and to define a series expansion which is convergent for the long wavelength limit $(K a \ll 1)$. The significant difference is that all wave modes are expanded as a series in $K a$, leaving dependence on the thermal wavenumber implicit in the coefficients. This avoids assumptions on the size of $L a$.

In the next section, the propagation of sound in fluids is considered in the context of a plane wave incident on a isolated spherical particle. The full ECAH method for the solution of the scattering problem is summarized and the perturbation expansion technique introduced. Sections 3 and 4 define the analytical forms of the wave potentials outside and inside the particle. Section 5 constructs the solution to the scattering problem in the perturbation method, showing the general solution and explicit results for the first few terms. Calculations are presented in section 6 to show that the method agrees with the full Rayleigh expansion method.

2. Sound fields in a fluid. The principles of sound propagation in homogeneous fluids are well documented, and only the most important results are given here. The equations of conservation of mass (continuity equation), momentum (Navier-Stokes equation), and energy, together with some thermodynamic relations, can be simplified by use of a velocity potential, $\phi$, such that

$$
\mathbf{u}=-\nabla \phi
$$

where $\mathbf{u}$ is the velocity of the fluid. The resulting biharmonic equation is further separated by defining two potentials, one for each of two types of wave mode (propagational and thermal). Propagational modes are the "usual" mode by which sound travels in a fluid. The thermal mode represents heat flow and is dissipative and therefore highly localized. There is an additional solution to the equations resulting from use of a vector potential, which corresponds to shear wave modes. Again these are dissipative with a very short decay length in fluids. In many practical applications of ultrasound, the shear wave modes resulting from scattering at dispersed particles are small. Hence for the subsequent analysis and in the previously published work (Harlen et al. [4], [5]), the vector potential solution is neglected. It was, however, included in the ECAH solutions. 
Periodic solutions for the wave potentials have a time dependence defined by a factor $e^{-i \omega t}$, where $\omega$ is the angular frequency, which results in two separate Helmholtz equations, one each for the propagational and thermal modes, thus:

$$
\left(\nabla^{2}+K^{2}\right) \varphi=0, \quad\left(\nabla^{2}+L^{2}\right) \psi=0 .
$$

The overall velocity potential in the fluid is the sum of the propagational $(\varphi)$ and thermal $(\psi)$ potentials.

The wavenumbers are given to a very good approximation in fluids by

$$
K=\frac{\omega}{v}\left(1+i \frac{(\gamma-1) \sigma \omega}{2 v^{2}}\right), \quad L=\left(\frac{\omega}{2 \sigma}\right)^{1 / 2}(1+i),
$$

where $\gamma$ is the ratio of specific heat capacities, $v$ the speed of sound, and $\sigma$ the thermal diffusivity, such that $\sigma=\tau / \rho C_{p}$, where $\tau$ is the thermal conductivity, $\rho$ is the density, and $C_{p}$ is the specific heat capacity at constant pressure.

The common expression for the wavenumber of the propagational mode has the form

$$
K=\frac{\omega}{v}+i \alpha
$$

where $\alpha$ is the attenuation. There are many absorption effects in fluids which are not accounted for in the classical thermal and fluid momentum equations used to derive the wavenumber in (3). Hence the measured attenuation should be used instead, as in (4).

The pressure and temperature fluctuations which result from the wave motions are related to the velocity potentials as follows:

$$
P=-i \omega \rho(\varphi+\psi), \quad T=\Gamma_{c} \varphi+\Gamma_{t} \psi,
$$

where the thermal factor for each wave mode is

$$
\Gamma_{c}=\frac{-i K^{2}(\gamma-1)}{\beta\left(\omega+i \gamma \sigma K^{2}\right)}, \quad \Gamma_{t}=\frac{-i L^{2}(\gamma-1)}{\beta\left(\omega+i \gamma \sigma L^{2}\right)} .
$$

Subscript $c$ is used to denote the compressional (or propagational) mode and $t$ the thermal mode. Note that these temperature factors were quoted incorrectly in the previous paper (Harlen et al. [5]).

A useful thermodynamic relation is

$$
\gamma-1=\frac{v^{2} \beta^{2} T_{0}}{C_{p}}
$$

where $\beta$ is the thermal expansivity and $T_{0}$ is the temperature of the system, not the small temperature changes caused by the wave motion.

2.1. Scattering of sound waves by particles. In order to calculate the ultrasound field produced by a dispersion of particles, it is first necessary to consider the effect on a sound wave of a single particle immersed in isolation in an infinite uniform fluid. The most relevant and simple system to study is that of a plane wave of angular frequency $\omega$ incident on a spherical particle of radius $a$. The fluid inside the particle has different physical properties and so will respond in a different way from the fluid surrounding it to the compression and rarefaction of the wave. Scattered waves of 
each mode are produced inside and outside the particle. At the surface of the particle certain boundary conditions must be met. These include the requirement that the boundary not be disrupted, i.e., that material immediately inside and outside of the particle move at the same speed, and that temperature and heat flow be continuous (the same either side of the interface). In terms of the wave potentials, the boundary conditions are as follows.

The normal velocity of fluid on both sides of the boundary must be equal to avoid formation of a void:

$$
\frac{\partial}{\partial r}\left(\varphi_{0}+\varphi+\psi\right)=\frac{\partial}{\partial r}\left(\varphi^{\prime}+\psi^{\prime}\right)
$$

The pressure must be equal on each side of the boundary:

$$
\varphi_{0}+\varphi+\psi=\hat{\rho}\left(\varphi^{\prime}+\psi^{\prime}\right) .
$$

The temperature must be equal on each side of the boundary:

$$
\Gamma_{c} \varphi_{0}+\Gamma_{c} \varphi+\Gamma_{t} \psi=\Gamma_{c}^{\prime} \varphi^{\prime}+\Gamma_{t}^{\prime} \psi^{\prime} .
$$

The heat flux must be equal on each side of the boundary:

$$
\Gamma_{c} \frac{\partial}{\partial r}\left(\varphi_{0}+\varphi\right)+\Gamma_{t} \frac{\partial \psi}{\partial r}=\hat{\tau}\left(\Gamma_{c}^{\prime} \frac{\partial \varphi^{\prime}}{\partial r}+\Gamma_{t}^{\prime} \frac{\partial \psi^{\prime}}{\partial r}\right),
$$

where primed quantities refer to the inside of the particle,

$$
\hat{\rho}=\frac{\rho^{\prime}}{\rho}, \quad \hat{\tau}=\frac{\tau^{\prime}}{\tau},
$$

and $\varphi_{0}$ is the potential of the incident wave.

The objective is to determine the amplitude and phase of the scattered propagational mode, which is the only part which is still nonnegligible at a significant distance from the particle (the thermal field having decayed to zero). Other published work is used to determine the wavenumber for a dispersion of particles, by a multiple scattering approach, to obtain the net effect of many particles. For the present work, the aim is to calculate the scattered wave amplitude.

In order to obtain the solution to the scattering problem, a general form for each wave mode must be proposed. The potentials of the scattered fields must be solutions of the appropriate Helmholtz equation (2), whether inside or outside the particle. In addition, the waves inside the particle must be defined at the origin (the center of the particle), and those outside the particle must satisfy the radiation condition. Finally, the boundary conditions at the surface of the particle must be satisfied. In sections 3 and 4 appropriate forms for the solutions are constructed to allow the scattering problem to be resolved.

2.2. ECAH method. The Epstein and Carhart method [2] for the scattering problem expanded the solutions of the Helmholtz equation in spherical coordinates. The solutions are Rayleigh series in the spherical harmonics, that is, a combined series in the spherical Bessel functions (for the radial dependence) and Legendre polynomials (for the angular dependence). The Bessel functions are chosen appropriately for the region in which the wave exists; in the continuous phase the solution must be defined at large distances, so the Hankel function $h_{n}$ is used, whereas inside the particle the 
solution must be defined at the origin, so the $j_{n}$ function is used. ECAH took the forms for each wave potential to be as follows:

$$
\begin{aligned}
& \varphi=\sum_{n=0}^{\infty} i^{n}(2 n+1) A_{n} h_{n}(K r) P_{n}(\cos \theta), \\
& \psi=\sum_{n=0}^{\infty} i^{n}(2 n+1) B_{n} h_{n}(L r) P_{n}(\cos \theta), \\
& \varphi^{\prime}=\sum_{n=0}^{\infty} i^{n}(2 n+1) A_{n}^{\prime} j_{n}\left(K^{\prime} r\right) P_{n}(\cos \theta), \\
& \psi^{\prime}=\sum_{n=0}^{\infty} i^{n}(2 n+1) B_{n}^{\prime} j_{n}\left(L^{\prime} r\right) P_{n}(\cos \theta) .
\end{aligned}
$$

Similarly the incident field (a plane wave) can be expressed as

$$
\varphi_{0}=\sum_{n=0}^{\infty} i^{n}(2 n+1) j_{n}(K r) P_{n}(\cos \theta)
$$

In the ECAH method, these functions and the relevant derivatives are directly evaluated at the surface of the particle and substituted into the set of boundary conditions. For a spherical particle of radius $a$ the Bessel functions must be determined at $r=a$. In the long wavelength limit, $|K a| \ll 1$ and $\left|K^{\prime} a\right| \ll 1$, the Hankel and Bessel functions can cause difficulties, and the difference in scale of the values appearing in the boundary equations results in an ill-conditioned matrix equation which must be solved.

In order to avoid these problems, a solution is sought which uses alternative forms for the wave potentials, both to avoid the direct use of the Bessel functions and to produce a direct solution not relying on a matrix inversion for its solution.

2.3. Kleinman method and Poincaré series. In the long wavelength limit, the condition $|K a| \ll 1$ applies. Kleinman developed a method for solving lowfrequency scattering problems (see Harlen et al. [4]) in which he expressed the potentials as a perturbation expansion, i.e., as a series in powers of $i K a$ :

$$
\phi=\sum_{m=0}^{\infty}(i K a)^{m} \phi_{m}
$$

where $\phi$ is one of the wave potentials. The series is known to converge rapidly, with an error bounded by $\mathrm{O}\left(|K a|^{m+1}\right)$ if the $m$ th order solution is used. Thus the problem becomes one of finding the solution to a set of potential functions. Although this may seem to increase the number of equations which must be solved, it avoids the ill-conditioned numerical calculation suffered by the ECAH approach and allows the series to be terminated with some confidence that an accurate result has been obtained. Further details of the method are given in Harlen et al. [4] and [5].

In the present work, all potentials are expanded as a series in $i K a$. The previous low-frequency work (Harlen et al. [4]) expanded each wave potential as a series in its appropriate wavenumber; for example, the thermal wave mode was defined as a series in powers of $L$. Later, the work on the short thermal wavelength region (geometric theory [5]) introduced combined power series, with positive powers of the 
propagational wavenumbers, and inverse powers of the thermal wavenumbers. In the frequency range considered in that work, the values of $|L a|$ and $\left|L^{\prime} a\right|$ were large, hence an inverse power series was appropriate. For example, for a scattered propagational mode potential,

$$
\phi=\sum_{n=0}^{\infty} \sum_{m=0}^{\infty} \frac{(i K a)^{n}}{(i L a)^{m}} \phi_{n m} .
$$

In order to achieve a method which is valid across the entire frequency range in the long wavelength limit, it is appropriate to use only a power series in $i K a$ since this value is by definition small over the entire range, $|K a| \ll 1$, thus assuring convergence of the series. Series in positive or inverse powers of $L a$ are limited in their scope because of the variation in magnitude of this parameter within the long wavelength region. Its value ranges from small at low frequency to very large at the upper frequency limit of the long wavelength region. Hence in the current work, the power series in $i K a$ is applied to each wave potential, with any dependence on other wavenumbers being implicit in the rest of the potential.

3. Solution forms outside the particle. In the continuous phase outside the particle, the solutions of the Helmholtz equation must also satisfy the radiation condition, which restricts its form at large distances from the particle. The Sommerfield radiation condition is as follows:

$$
\lim _{r \rightarrow \infty}\left[r\left(\frac{\partial \phi}{\partial r}-i k \phi\right)\right]=0
$$

In physical terms, the condition means that there is no energy radiating inwards from infinity. Thus the solution appears as an outgoing spherical wave at large distances from the particle (cf. Colton and Kress [6, p. 21]).

The spherical Hankel function used in the ECAH method is one such solution, each $h_{n}(k r)$ including a factor $e^{i k r} / r$ which represents a spherical wave. However, radiating solutions to the Helmholtz equation are not regular at infinity, and it is the exponential part of the function which caused numerical difficulties at large arguments (for the thermal waves).

In general terms, the form of the solution is

$$
\phi=\frac{e^{i k r}}{r} \tilde{\phi}=\frac{e^{i k r}}{r} \sum_{l=0}^{\infty} \frac{f_{l}(\vartheta, \Omega)}{r^{l}},
$$

where $f_{l}$ is the angular dependence (Harlen et al. [4]). The function $\tilde{\phi}$ does not suffer from the mathematical difficulties of the overall potential $\phi$, and is regular at infinity.

Following the previous method (Harlen et al. [4]), it is therefore appropriate to introduce new potential functions, $\tilde{\varphi}$ and $\tilde{\psi}$, for the propagational and thermal modes, respectively, outside the particle such that

$$
\begin{aligned}
& \varphi=e^{i K(r-a)} \tilde{\varphi}, \\
& \psi=e^{i L(r-a)} \tilde{\psi} .
\end{aligned}
$$

The exponential spherical-wave factors have been explicitly taken out, so that the remaining functions $\tilde{\varphi}$ and $\tilde{\psi}$ are regular and differentiable. In addition when applying 
boundary conditions for a spherical particle at $r=a$ the exponential factors do not contribute.

Continuing the Kleinman approach, the next step is to express the new potential functions as a series in $i K a$ (see previous section), thus

$$
(\tilde{\varphi}, \tilde{\psi})=\sum_{m}(i K a)^{m}\left(\tilde{\varphi}_{m}, \tilde{\psi}_{m}\right)
$$

3.1. Propagational mode. Using the spherical harmonic solutions to the Helmholtz equation, the partial fields for the propagational mode can then be written as

$$
\tilde{\varphi}_{m}=\sum_{n=0}^{\infty} \sum_{j=0} A_{n m j} \cdot \frac{r^{j}}{a^{j}} \cdot \frac{a^{n+1}}{r^{n+1}} \cdot P_{n}(\cos \vartheta) .
$$

This form of the solution is suggested by the results of the LFPST method (Harlen et al. [4]), although it is expressed here as a general series in $r$. The full wave potential can be constructed using (21) and (19). The Helmholtz equation (2) can then be shown to relate the potentials of consecutive order $m$ by the equation

$$
\nabla^{2} \tilde{\varphi}_{m}=-\frac{2}{a r} \frac{\partial}{\partial r}\left(r \tilde{\varphi}_{m-1}\right)
$$

By substituting the general solution (22) into this form of the Helmholtz equation, and matching powers of ( $i K a)$ for each (spherical harmonic) order $n$, it can be shown that the coefficients are related by the following recurrence relation:

$$
A_{n, m, j}=-\frac{2(j-1-n)}{j(j-1-2 n)} A_{n, m-1, j-1} \text { for } j \geq 1
$$

Thus coefficients for the potential of order $m$ are related to those for the previous order. Only the $j=0$ coefficient remains to be solved from the boundary equations. By definition, all coefficients for orders $m<0$ are zero. Note that the coefficients are zero for $j \geq n+1$, and hence the coefficients may be nonzero up to and including $j=n$, i.e., it is a finite series. The solution for the lowest orders (see section 5.6) demonstrates that, excepting $n=0$, the first (in $m$ ) nonzero coefficient is for $m=n$, which implies that at larger orders $m$ the last nonzero term in the $j$-series will be for $j=m-n$. The result also shows that (22) does give a solution of the Helmholtz equation. Although the propagational mode solution (22) does not appear to have the same form as that required for a radiating solution (18), it is clear that since the coefficients are nonzero only for $j \leq n$, each term in $l$ (18) includes contributions from different $m, n$, and $j$ combinations, which together give the angular dependence $f_{l}$.

The ECAH method expresses the propagational scattered wave in terms of the spherical Hankel functions $h_{n}(K r)$. Our result is not simply a power series expansion in $K r$ of the Hankel function. This is because the wave potential has been written as a power series in $i K a$, which removes all the $K$-dependence, leaving a series in $r$, whose coefficients are to be determined. Contributions from different orders $m$ make up the overall potential series in $K r$.

3.2. Thermal mode. In the ECAH method, the thermal wave potential in the continuous phase was based on the spherical Hankel function $h_{n}(L r)$. Since the perturbation series expansion (21) is taken in powers of $(i K a)$, rather than in powers 
of $L$, the thermal potential can be expressed simply using the series expansion of the spherical Hankel function. Thus

$$
h_{n}(x)=e^{i x} \sum_{j=1}^{n+1} \frac{h_{n j}}{x^{j}}
$$

(see the appendix for the factors $h_{n j}$ ), so that the thermal wave potential takes the form

$$
\tilde{\psi}_{m}=\sum_{n=0}^{\infty} \sum_{j=1}^{n+1} B_{n m} \cdot \frac{h_{n j}}{(L r)^{j}} \cdot P_{n}(\cos \vartheta) .
$$

All factors of $L$ are taken implicitly as part of the potential function. The spherical Hankel function, in fact, results in a finite series in inverse powers of $L r$, whose coefficients are known. These are all included in the appropriate term in the series in powers of $(i K a)^{m}$.

3.3. The incident wave. The incident wave is a plane wave and can be expressed as a series of spherical harmonics, as in the ECAH method (see (14)). In order to follow the same method as used for the other waves, the spherical Bessel function can be expanded as a power series in $(i K a)$, using the power series expansion of the spherical Bessel function

$$
j_{n}(x)=2^{n} x^{n} \sum_{s=0}^{\infty} \frac{(-1)^{s}(s+n) !}{s !(2 s+2 n+1) !} x^{2 s}
$$

(Arfken [7, p. 625]).

Thus the plane wave can be written

$$
\varphi_{0}=\sum_{n=0}^{\infty} \sum_{s=0}^{\infty}(i K a)^{n+2 s}\left(\frac{r}{a}\right)^{n+2 s} F_{n}(s) P_{n}(\cos \vartheta),
$$

where

$$
F_{n}(s)=\frac{2^{n}(2 n+1)(s+n) !}{s !(2 s+2 n+1) !},
$$

where $n$ and $s$ are nonnegative integers ( $F$ is zero otherwise). For purposes of numerical calculation, the factorial functions suffer from overflow for all but very low orders $(n, s)$. The following recurrence relations can be used for accurate calculation:

$$
\begin{aligned}
F_{0}(0) & =1, \\
\frac{F_{n}(0)}{F_{n-1}(0)}=\frac{1}{(2 n-1)} \text { for } n & \geq 1, \\
\frac{F_{n}(s)}{F_{n}(s-1)}=\frac{1}{2 s(2 n+2 s+1)} \text { for } s & \geq 1 .
\end{aligned}
$$

In the low-frequency scattering method (Harlen et al. [4]), the incident field was included in the form of (14), with the $j_{n}(K r)$ function retained. Thus the contribution of the incident field was included entirely in the zeroth and first order terms of the perturbation series in $(i K a)$. The later work for the higher frequency region 
(Harlen et al. [5]) expressed the incident field through the power series expansion of the exponential form:

$$
e^{i K z}=e^{i K r \cos \theta}=\sum_{n=0}^{\infty} \frac{(i K a \cos \theta)^{n}}{n !} .
$$

However, this requires that each of the powers of $\cos \theta$ be expressed in terms of the associated Legendre polynomials $P_{n}(\cos \theta)$ which appear in the other wave potentials in order to match the angular dependence in the boundary conditions. Hence the expansion used here, (28), appears to be most consistent with the method, since the powers of $i K a$ and the angular dependence $P_{n}(\cos \theta)$ can be matched directly in the boundary equations.

4. Solution forms inside the particle. Within the particle or droplet, the solutions to the Helmholtz equation need not satisfy the radiation condition, since the waves are in a bounded region. However, the solutions must be defined at the origin $(r=0)$. In spherical coordinates the appropriate solutions for the radial part of the potential are the spherical Bessel functions, $j_{n}$, rather than the spherical Hankel functions, $h_{n}$, which are not defined at the origin. When the boundary conditions are applied, the Bessel function for the thermal wave must be evaluated for an argument $L^{\prime} a$ which can have a large imaginary component in the frequency range of interest. Hence, it is again desirable to avoid the use of Bessel functions.

4.1. Propagational mode. There are many different ways of expressing the spherical Bessel functions $j_{n}$-for example, as an infinite power series or as a combination of trigonometric functions sin and $\cos$. For the propagational mode inside the particle, the power series form can be used, since the value $K^{\prime} a$ (which is the argument of the function used in the boundary equations) is small in the long wavelength region. First, applying the perturbation series expansion as

$$
\varphi^{\prime}=\sum_{m}(i K a)^{m} \varphi_{m}^{\prime}
$$

and then expressing the potential as a series in powers of $r$ gives

$$
\varphi_{m}^{\prime}=\sum_{n=0}^{\infty} \sum_{j=0} A_{n m j}^{\prime} \cdot \frac{r^{j}}{a^{j}} \cdot \frac{r^{n}}{a^{n}} \cdot P_{n}(\cos \vartheta) .
$$

The Helmholtz equation (2) can again be used with (34) to relate potentials to those of a different order, and thus

$$
\nabla^{2} \varphi_{m}^{\prime}=\frac{\hat{c}}{a^{2}} \varphi_{m-2}^{\prime}
$$

where

$$
\hat{c}=\frac{K^{\prime 2}}{K^{2}},
$$

which is frequency independent to a very good approximation. Substituting the general solution, (35), into (36) and matching powers of $i K a$ as before results in the following recurrence relation for the coefficients:

$$
\begin{aligned}
A_{n, m, j}^{\prime} & =\frac{\hat{c}}{j(2 n+j+1)} A_{n, m-2, j-2}^{\prime} \text { for } j \geq 2, \\
A_{n, m, j}^{\prime} & =0 \text { for } j=1 \text { and all odd values of } j .
\end{aligned}
$$


As in the case of the propagational mode in the continuous phase, the above equations show that the coefficients $A_{n, m, j}^{\prime}$ for order $m$ can be calculated from those of a previous order, given that all coefficients for $m<0$ are zero. Only the $j=0$ coefficient remains to be determined from the boundary conditions. In this case the limit of the series is determined by the number of nonzero coefficients for the previous order, producing an expanding triangle of coefficients. The solution of the boundary conditions shows that the first nonzero coefficient is for $n=m, j=0$, so that the limit of the series in $j$ would be $j=m-n$, where $m>n$.

4.2. Thermal mode. For the thermal wave, the argument of the function at the boundary, $L^{\prime} a$, may be small or very large, depending on the frequency. The usual power series expansion of $j_{n}$ would be inappropriate at large arguments. Similarly an infinite inverse power series in $L^{\prime} r$, as used by Harlen et al. [5], would be unsuitable at small arguments and is not defined at the origin. Hence, either form is restricted in its frequency range.

The trigonometric form of the Bessel function $j_{n}$ (e.g., $j_{0}(x)=\sin x / x$ ) can be modified by expressing the trigonometric functions as a sum or difference of two exponential terms $e^{i x}$ and $e^{-i x}$, and thus

$$
j_{n}(x)=e^{i x} \sum_{j=1}^{n+1} \frac{j_{n j+}}{x^{j}}-e^{-i x} \sum_{j=1}^{n+1} \frac{j_{n j-}}{x^{j}}
$$

(see the appendix for the coefficients).

Hence, the wave potential for the thermal wave in the particle could be written as a sum of modified outward and inward spherical waves. Arfken [7, p. 627] states that " $j_{n}(x)$ and $n_{n}(x)$ are appropriate for a description of standing spherical waves; $h_{n}^{1}(x)$ and $h_{n}^{2}(x)$ correspond to traveling spherical waves." A standing wave results from a superposition of traveling waves in opposite directions.

Hence the thermal wave inside the particle can be written as

$$
\psi^{\prime}=e^{i L^{\prime}(r-a)} \tilde{\psi}_{+}^{\prime}-e^{-i L^{\prime}(r-a)} \tilde{\psi}_{-}^{\prime} .
$$

Following the previous perturbation series method with each of the new wave potentials,

$$
\left\{\tilde{\psi}_{+}^{\prime}, \tilde{\psi}_{-}^{\prime}\right\}=\sum_{m}(i K a)^{m}\left\{\tilde{\psi}_{+m}^{\prime}, \tilde{\psi}_{-m}^{\prime}\right\} .
$$

And each of the terms has the usual angular dependence; thus

$$
\begin{gathered}
\tilde{\psi}_{+m}^{\prime}=\sum_{n=0}^{\infty} \sum_{j=1}^{n+1} B_{n m}^{\prime} \cdot e^{2 i L^{\prime} a} \cdot \frac{j_{n j+}}{\left(L^{\prime} r\right)^{j}} \cdot P_{n}(\cos \vartheta), \\
\tilde{\psi}_{-m}^{\prime}=\sum_{n=0}^{\infty} \sum_{j=1}^{n+1} B_{n m}^{\prime} \cdot \frac{j_{n j-}}{\left(L^{\prime} r\right)^{j}} \cdot P_{n}(\cos \vartheta) .
\end{gathered}
$$

The factor $e^{2 i L^{\prime} a}$ results from the condition that the potential be defined at the origin. All inverse powers of $r$ must cancel at the origin, leaving only a single term from $n=0$.

The previous work on scattering at the high-frequency end of the long wavelength limit (Harlen et al. [5]) used only the second of the two terms given in (41). When a series solution is used in powers of $L^{\prime} a$ the only solution for the zeroth order term is $a / r$ (Harlen et al. [5, equation (4.9)]), which results in a function which is not defined at the origin. The result given above avoids this problem. 


\section{Construction of the solution.}

5.1. Pressure and temperature factors. When applying the boundary conditions, terms in the same powers of $i K a$ must be matched, as must the angular dependence $P_{n}(\cos \theta)$. For consistency, any other parameters which appear in the boundary conditions must be expressed as the appropriate power of $i K a$. The pressure and temperature changes caused by the different waves were defined in (5). The pressure is related to the wave potential by a factor which includes the frequency and density. Since the frequency is the same for all wave forms, this factor will cancel from the relevant boundary equation. However, the thermal factors (equation (6)) have different frequency dependence for the different wave modes, and these need to be defined in relation to powers of $i K a$.

For the propagational modes, the thermal factors can be simplified by using the relation

$$
\left|\frac{K^{2}}{L^{2}}\right| \approx \frac{\omega \sigma}{v^{2}} \ll 1
$$

The approximation is not limited in frequency range, but relies on the small value of $\omega \sigma / v^{2}$ which is of order $10^{-5}$ at $100 \mathrm{MHz}$ in water at $30^{\circ} \mathrm{C}$, so that

$$
\Gamma_{c}=\frac{-i K^{2}(\gamma-1)}{\beta\left(\omega+i \gamma \sigma K^{2}\right)} \approx \frac{K^{2}(\gamma-1)}{\beta \sigma L^{2}}
$$

and

$$
\Gamma_{c}^{\prime}=\frac{-i K^{\prime 2}\left(\gamma^{\prime}-1\right)}{\beta^{\prime}\left(\omega+i \gamma^{\prime} \sigma^{\prime} K^{\prime 2}\right)} \approx \frac{\hat{c} K^{2}\left(\gamma^{\prime}-1\right)}{\beta^{\prime} \sigma^{\prime} L^{\prime 2}} .
$$

The dependence on $L$ is left implicit, whereas the power series in $(i K a)$ requires explicit consideration of powers of $K$. Hence the $K$-dependence of thermal factors are expressed by two new parameters, and thus

$$
\Gamma_{c}=(i K a)^{2} g_{c}, \quad \Gamma_{c}^{\prime}=(i K a)^{2} g_{c}^{\prime} .
$$

The thermal factors for the thermal wave modes can be simplified,

$$
\Gamma_{t}=\frac{-i L^{2}(\gamma-1)}{\beta\left(\omega+i \gamma \sigma L^{2}\right)} \approx-\frac{1}{\beta \sigma},
$$

and similarly in the dispersed phase. The temperature factors for the thermal waves can therefore be seen to be approximately independent of frequency, and hence independent of $K$, which is the power series being used.

5.2. Definitions. The application of the boundary conditions leads to some complicated equations, which can be made easier to read by using some further symbols to define collections of terms. In addition, in numerical calculation, greater accuracy is achieved (avoiding subtraction of nearly equal terms) by using the recurrence relation (24) to write

$$
\sum_{j=0} A_{n, m-1, j}+\sum_{j=0}^{m-n} j A_{n m j}=\sum_{j=1}^{m-n}-\frac{j}{(j-2 n)} A_{n, m-1, j}+\delta_{n 0} A_{n, m-1,0},
$$


where $\delta_{n 0}$ is a Kronecker delta. The second term on the right-hand side which affects only the $n=0$ results was omitted in the reported LFPST solution (Harlen et al. [4]).

Other symbols are defined as follows:

$$
\begin{gathered}
S_{h}=\sum_{j=1}^{n+1} \frac{h_{n j}}{(L a)^{j}}, \\
S_{d h}=\sum_{j=1}^{n+1} \frac{(i L a-j) h_{n j}}{(L a)^{j}}, \\
S_{j}=e^{2 i L^{\prime} a} \sum_{j=1}^{n+1} \frac{j_{n j+}}{\left(L^{\prime} a\right)^{j}}-\sum_{j=1}^{n+1} \frac{j_{n j-}}{\left(L^{\prime} a\right)^{j}}, \\
S_{d j}=e^{2 i L^{\prime} a} \sum_{j=1}^{n+1} \frac{\left(i L^{\prime} a-j\right) j_{n j+}}{\left(L^{\prime} a\right)^{j}}+\sum_{j=1}^{n+1} \frac{\left(i L^{\prime} a+j\right) j_{n j-}}{\left(L^{\prime} a\right)^{j}}, \\
S_{A, m-s}=\sum_{j=0}^{n} A_{n, m-s, j} \text { for } s=1,2,3, \quad S_{A, m}=\sum_{j=1}^{n} A_{n, m, j}, \\
S_{j A^{\prime}, m-s}=\sum_{j=1}^{m-n} j A_{n, m-s, j}^{\prime} \text { for } s=0,1,2 . \\
\sum_{A^{\prime}, m-s}^{n}=\sum_{j=0}^{m-n} \frac{j}{(j-2 n)} A_{n, m-s, j}^{\prime}+\delta_{n 0} A_{n, m-s, 0} \text { for } s=0,1,2,3, \\
S_{A^{\prime}, m}=\sum_{j=1}^{m-n} A_{n, m, j}^{\prime},
\end{gathered}
$$

5.3. Boundary conditions. Having defined the wave potentials in a consistent form, as perturbation series in powers of $i K a$, and with the Legendre polynomials defining the angular dependence, the boundary conditions can now be applied at the surface of the spherical particle, $r=a$. Each boundary equation consists of summations over orders $n, m$. The spherical harmonic terms which define the angular dependence are independent and hence must be matched - so all terms in the same $n$ must be matched. In addition, terms in powers of $(i K a)^{m}$ are matched on each side of the equation, which may arise from various orders of $m$. If each order $m$ is determined in turn, all coefficients for previous orders, e.g., $m-1$, are already known. In addition, the propagational mode coefficients for order $m$ for $j \geq 1$ can be calculated from the previous order results (see (24) and (38)). Hence, the boundary equations for the $n, m$ th order include four unknowns:

$$
A_{n m 0}, A_{n m 0}^{\prime}, B_{n m}, B_{n m}^{\prime}
$$


The four boundary conditions (8)-(11) result in the equations below:

$m F_{n}\left(\frac{m-n}{2}\right)+\sum_{j=0}^{m-1-n} A_{n, m-1, j}+\sum_{j=1}^{m-n}(j-n-1) A_{n m j}-(n+1) A_{n m 0}+S_{d h} B_{n m}$

$$
=\sum_{j=1}^{m-n}(j+n) A_{n m j}^{\prime}+n A_{n m 0}^{\prime}+S_{d j} B_{n m}^{\prime},
$$

$$
F_{n}\left(\frac{m-n}{2}\right)+\sum_{j=1}^{m-n} A_{n m j}+A_{n m 0}+S_{h} B_{n m}
$$

$$
\begin{aligned}
g_{c} F_{n}\left(\frac{m-n-2}{2}\right) & +g_{c} \sum_{j=0}^{m-2-n} A_{n, m-2, j}+\Gamma_{t} S_{h} B_{n m} \\
& =g_{c}^{\prime} \sum_{j=0}^{m-2-n} A_{n, m-2, j}^{\prime}+\Gamma_{t}^{\prime} S_{j} B_{n m}^{\prime}
\end{aligned}
$$

$$
\begin{array}{r}
g_{c}(m-2) F_{n}\left(\frac{m-n-2}{2}\right)+g_{c} \sum_{j=0}^{m-3-n} A_{n, m-3, j} \\
\quad+g_{c} \sum_{j=0}^{m-2-n}(j-n-1) A_{n, m-2, j}+\Gamma_{t} S_{d h} B_{n m} \\
=\hat{\tau} g_{c}^{\prime} \sum_{j=0}^{m-2-n}(j+n) A_{n, m-2, j}^{\prime}+\hat{\tau} \Gamma_{t}^{\prime} S_{d j} B_{n m}^{\prime} .
\end{array}
$$

5.4. Solution. The solution proceeds by stepping through the orders of $m$ starting at $m=0$. All coefficients are zero for $m<0$. For each $m$ the recurrence relations are used to derive any nonzero propagational mode coefficients for order $m$ (equations (24) and (38)). The two thermal boundary conditions, (61) and (62), for the $n, m$ th order include only the unknown thermal coefficients; other terms, being from previous orders $m-2$ and $m-3$, are already known. Hence (61) and (62) can be solved for the thermal coefficients $B_{n m}$ and $B_{n m}^{\prime}$. These can be substituted into the other boundary equations, (59) and (60), in order to determine the remaining propagational mode coefficients $A_{n m 0}, A_{n m 0}^{\prime}$.

The thermal coefficients for $n, m$ are

$$
\begin{aligned}
B_{n m}= & {\left[-g_{c}\left(\hat{\tau} S_{d j}-(m-2) S_{j}\right) F_{n}((m-n-2) / 2)\right.} \\
& -g_{c}\left\{\left(\hat{\tau} S_{d j}+(n+1) S_{j}\right) S_{A, m-2}-S_{j} S_{j A, m-3}\right\} \\
& \left.+\hat{\tau} g_{c}^{\prime}\left\{\left(S_{d j}-n S_{j}\right) S_{A^{\prime}, m-2}-S_{j} S_{j A^{\prime}, m-2}\right\}\right] / \Gamma_{t}\left(\hat{\tau} S_{d j} S_{h}-S_{j} S_{d h}\right),
\end{aligned}
$$




$$
\begin{aligned}
B_{n m}^{\prime}= & {\left[-g_{c}\left(S_{d h}-(m-2) S_{h}\right) F_{n}((m-n-2) / 2)\right.} \\
& -g_{c}\left\{\left(S_{d h}+(n+1) S_{h}\right) S_{A, m-2}-S_{h} S_{j A, m-3}\right\} \\
& \left.+g_{c}^{\prime}\left\{\left(S_{d h}-n \hat{\tau} S_{h}\right) S_{A^{\prime}, m-2}-\hat{\tau} S_{h} S_{j A^{\prime}, m-2}\right\}\right] / \Gamma_{t}^{\prime}\left(\hat{\tau} S_{d j} S_{h}-S_{j} S_{d h}\right) .
\end{aligned}
$$

Substituting into the boundary conditions, (59) and (60), thus gives

$$
\begin{aligned}
A_{n m 0}= & {\left[(m \hat{\rho}-n) F_{n}((m-n) / 2)\right.} \\
& -(n+(n+1) \hat{\rho}) S_{A, m}+\hat{\rho}\left(S_{j A, m-1}-S_{j A^{\prime}, m}\right) \\
& \left.+\left(\hat{\rho} S_{d h}-n S_{h}\right) B_{n m}-\hat{\rho}\left(S_{d j}-n S_{j}\right) B_{n m}^{\prime}\right] /[n+(n+1) \hat{\rho}], \\
A_{n m 0}^{\prime}= & {\left[(m+n+1) F_{n}((m-n) / 2)\right.} \\
& -(n+(n+1) \hat{\rho}) S_{A^{\prime}, m}+\left(S_{j A, m-1}-S_{j A^{\prime}, m}\right) \\
& \left.+\left(S_{d h}+(n+1) S_{h}\right) B_{n m}-\left(S_{d j}+(n+1) \hat{\rho} S_{j}\right) B_{n m}^{\prime}\right] /[n+(n+1) \hat{\rho}] .
\end{aligned}
$$

Since the thermal boundary equations (61) and (62) include terms in order $m-2$ and lower orders, this implies that the first nonzero thermal field contribution is at second order in $i K a$.

5.5. Multiple scattering. For practical application of the scattering results, it is necessary to relate the scattering from a single particle to the wavenumber (and corresponding velocity and attenuation) of a dispersion of such particles. The commonly used formulation for multiple scattering is that of Lloyd and Berry [8], whose result was derived in a different way by Waterman and Truell and later works [9], [10]. Taking the limiting form of the solution, (18), in the far field as $r$ approaches infinity, the scattered field takes the form

$$
\varphi \rightarrow \frac{e^{i K r}}{r} f(\theta)
$$

In terms of the Legendre polynomials,

$$
f(\theta)=\frac{1}{i K} \sum_{n=0}^{\infty}(2 n+1) T_{n} P_{n}(\cos \theta) .
$$

The scattered propagational field, combining (19), (21), and (22), has the form

$$
\varphi=\sum_{m=0}^{\infty} \sum_{n=0}^{\infty} \sum_{j=0} A_{n m j}(i K a)^{m} e^{i K(r-a)} \cdot \frac{r^{j}}{a^{j}} \cdot \frac{a^{n+1}}{r^{n+1}} \cdot P_{n}(\cos \vartheta),
$$

from which the far field coefficient $T_{n}$ is

$$
T_{n}=\frac{e^{-i K a}}{(2 n+1)} \sum_{m=0}^{\infty}(i K a)^{m+1} A_{n m n}
$$

showing that only the terms $j=n$ contribute in the far field. For each spherical harmonic $n$ (except for $n=0$ ), the first nonzero term for the $j=0$ coefficients (from the boundary equations) is for $m=n$ (see, for example, the results in Table 1). 
Following the recurrence relation (equation (24)) through the orders $m$ shows that the first nonzero term appearing in the far field (i.e., for $j=n$ ) will be at order $m=2 n$. So for the $n=1$ far field coefficient, $T_{1}$, the first contribution results from $A_{121}$; for $n=2$ the first nonzero term in $T_{2}$ corresponds to $m=4$, i.e., $A_{242}$. It is very important to include sufficient orders $m \geq 2 n$ when an accurate solution for the $n$th order far field coefficient is required.

The multiple scattering result for the wavenumber of the dispersion, $B$, is

$$
\begin{aligned}
& \left(\frac{B}{K}\right)^{2}=1+\frac{3 \phi}{K^{2} a^{3}} f(0) \\
& +\frac{9 \phi^{2}}{4 K^{4} a^{6}}\left(f^{2}(\pi)-f^{2}(0)-\int_{0}^{\pi} d \theta \frac{1}{\sin (\theta / 2)}\left(\frac{d}{d \theta} f^{2}(\theta)\right)\right),
\end{aligned}
$$

which to second order gives

$$
\begin{aligned}
& \left(\frac{B}{K}\right)^{2}=1-\frac{3 i \phi}{K^{3} a^{3}}\left(T_{0}+3 T_{1}+5 T_{2}\right) \\
& -\frac{27 \phi^{2}}{K^{6} a^{6}}\left(T_{0} T_{1}+\frac{10}{3} T_{0} T_{2}+2 T_{1}^{2}+11 T_{1} T_{2}+\frac{230}{21} T_{2}^{2}\right) .
\end{aligned}
$$

Note that here the symbol $\phi$ refers to the volume fraction of the dispersed particles.

5.6. Explicit solutions for low orders. In order to demonstrate the method of solution and to derive explicit solutions which may be used instead of the general solution, the results are here derived for low orders of $n$ and $m$. The following parameters are those which are used to obtain the low order solutions:

For $n=0$

$$
\begin{gathered}
h_{01}=-i, \\
S_{h}=-i /(L a), \quad S_{d h}=-i(i L a-1) /(L a), \\
j_{01+}=j_{01-}=1 /(2 i), \\
S_{j}=\left(e^{2 i L^{\prime} a}-1\right) /\left(2 i L^{\prime} a\right), \\
S_{d j}=\left\{i L^{\prime} a\left(e^{2 i L^{\prime} a}+1\right)-\left(e^{2 i L^{\prime} a}-1\right)\right\} /\left(2 i L^{\prime} a\right), \\
F_{0}(0)=1, \quad F_{0}(1)=1 / 6,
\end{gathered}
$$

and for $n=1$

$$
F_{1}(0)=1 .
$$

All the nonzero coefficients are given in Table 1 for orders $n \leq 2$ and $m \leq 2$. The method for obtaining these solutions is summarized below. 
TABLE 1

Explicit solutions for scattering coefficients at low orders.

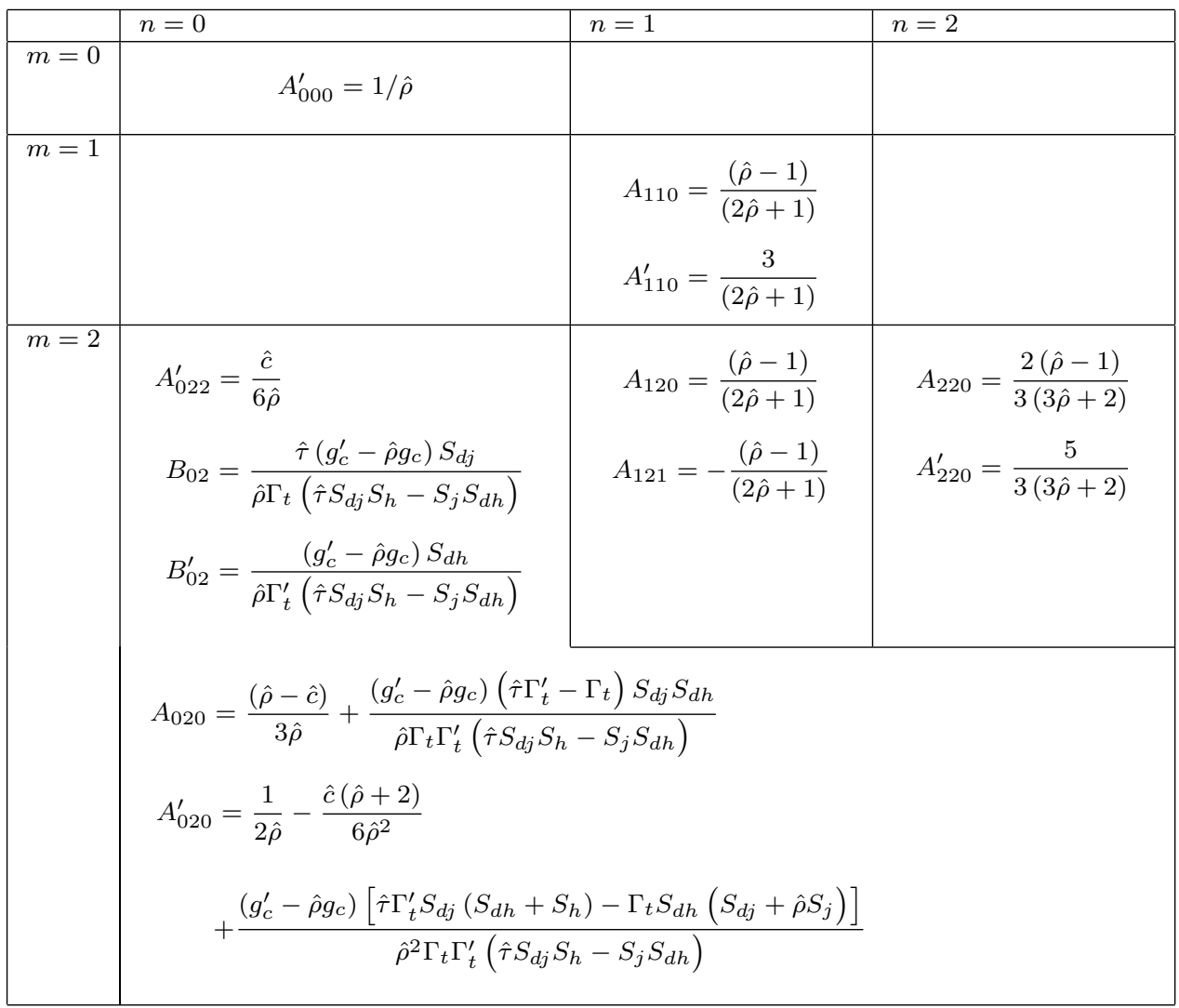

By definition, all coefficients for orders $m<0$ are zero; hence the recurrence relations (equations (24) and (38)) show that all propagational mode coefficients are zero for $j>0$. There is no incident field contribution to the thermal boundary conditions (equations (61) and (62)) (since $s=(m-n-2) / 2=-1$ ), so the thermal coefficients are zero:

$$
B_{00}=B_{00}^{\prime}=0
$$

The velocity and pressure boundary conditions (equations (59) and (60)) include a nonzero contribution from the incident field, such that the zeroth order of the incident field affects the zeroth order propagational mode. The resulting coefficients for the propagational modes are

$$
A_{000}=0, \quad A_{000}^{\prime}=1 / \hat{\rho} .
$$

For $m=1$ the incident field makes no contribution at the boundary (since the arguments $s=(m-n-2) / 2$ or $s=(m-n) / 2$ are noninteger). No nonzero coefficients are found from the recurrence relations, so again the thermal field is zero. The propagational coefficients are also zero in this case (by substitution in the boundary equations):

$$
A_{010}=A_{010}^{\prime}=0 .
$$


For $m=2$, the recurrence relations now give a nonzero coefficient, $A_{022}^{\prime}$, resulting from the $A_{000}^{\prime}$ coefficients, as shown in the table.

The summations over coefficients also now have nonzero terms:

$$
S_{A^{\prime}, m}=\frac{\hat{c}}{6 \hat{\rho}}, \quad S_{j A^{\prime}, m}=\frac{\hat{c}}{3 \hat{\rho}}, \quad S_{A^{\prime}, m-2}=\frac{1}{\hat{\rho}} .
$$

All coefficients $(j>0)$ of the continuous phase propagational mode are again zero.

The incident field contributes both to the thermal boundary conditions and to the velocity and pressure conditions producing the first nonzero thermal coefficients, $B_{02}, B_{02}^{\prime}$ (Table 1). These results are consistent with the results in the geometric theory paper (Harlen et al. [5]) for large values of La. The LFPST paper (Harlen et al. [4]) did not assign the incident field to the different orders of $K a$, instead assigning it all to the zeroth and first order terms, so analytical comparison is not possible. The propagational coefficients, $A_{020}, A_{020}^{\prime}$ (Table 1) are found by substituting the thermal coefficients into the velocity and pressure boundary equations. For higher orders in $n$ the process is exactly the same, but there are no thermal contributions up to order $m=2$, resulting in coefficients which depend only on density.

To obtain the velocity and attenuation of a dispersion using the coefficients up to second order, the relevant far field coefficients, $T_{n}$, must be determined. Equation (70) shows that only coefficients for $j=n$ contribute to each $T_{n}$. Thus the far field coefficients to second order in $m$ are

$$
T_{0}=e^{-i K a}(i K a)^{3} A_{020}, \quad T_{1}=\frac{e^{-i K a}}{3}(i K a)^{3} A_{121}, \quad T_{2}=0 .
$$

Substituting these coefficients into the equation for the wavenumber of the dispersion, $B$ (equation (72)), gives

$$
\left(\frac{B}{K}\right)^{2}=1-3 \phi e^{-i K a}\left(A_{020}+A_{121}\right)+3 \phi^{2} e^{-2 i K a}\left(3 A_{020} A_{121}+2 A_{121}^{2}\right) \text {. }
$$

Since the exponential factors are near unity ( $K a$ being small), and the coefficient $A_{121}$ depends only on density, the particle size and frequency dependence appear almost entirely through the $A_{020}$ coefficient. It is the parameters $S_{h}, S_{d h}, S_{j}, S_{d j}$ (equations (74) and (76)) which define the dependence on particle size and frequency through the relationship between the thermal wavelengths and the particle size, expressed by the parameters $L a$ and $L^{\prime} a$. Thus we have an analytical result for the wavenumber of the dispersion which is valid over the entire long wavelength region, and is simple enough to be calculated in a standard spreadsheet. It is, of course, an approximate result, but, as will be demonstrated in the next section, it is a good approximation unless the parameter $K a>0.01$. Visco-inertial scattering has not been included in the present theory, so for dispersions with a large density difference between the two components, the results will not be as accurate.

6. Results. Calculations have been carried out using MATLAB for a model system of sunflower oil in water at $30^{\circ} \mathrm{C}$. The calculations are straightforward, and take only a few seconds to complete a spectrum of 50 frequency values. The physical properties of the two components are given in Table 2. A particle diameter of $1 \mu \mathrm{m}$ was chosen so that a complete range of thermal wavelengths could be covered within the long wavelength limit. The concentration (by volume) was $20 \%$. The attenuation of each material was not included in the calculation, so the attenuation determined by 
TABLE 2

Physical properties of sunflower oil and water at $30^{\circ} \mathrm{C}$.

\begin{tabular}{|l|l|l|}
\hline & Water & Sunflower oil \\
\hline Ultrasound velocity / $\mathrm{m} \mathrm{s}^{-1}$ & 1509.1 & 1437.9 \\
\hline Density / $\mathrm{kg} \mathrm{m}^{-3}$ & 995.7 & 912.9 \\
\hline Thermal expansivity / $\mathrm{K}^{-1}$ & 0.00030 & 0.00073 \\
\hline Specific heat capacity / $\mathrm{J} \mathrm{kg}^{-1} \mathrm{~K}^{-1}$ & 4178.2 & 1980.0 \\
\hline Thermal conductivity / $\mathrm{W} \mathrm{m}^{-1} \mathrm{~K}^{-1}$ & 0.603 & 0.17 \\
\hline
\end{tabular}

$\mid \mathrm{Lal}$

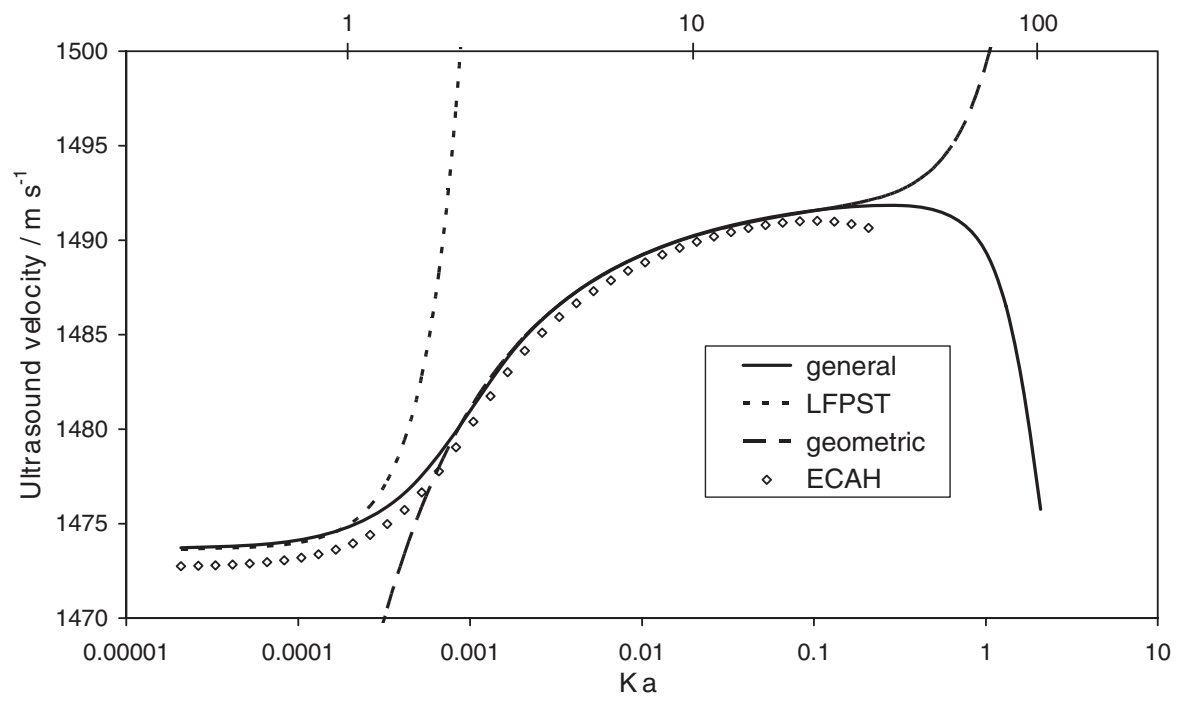

FIG. 1. Ultrasound velocity as a function of the parameter Ka for $20 \%$ sunflower oil in water at $30^{\circ} \mathrm{C}$ with a particle diameter of $1 \mu \mathrm{m}$. Four different calculation methods are shown: the "general" method presented in this paper, the LFPST method (Harlen et al. [4]), the geometric theory method (Harlen et al. [5]), and the ECAH method (Epstein and Carhart [2]). The thermal parameter $|L a|$ is shown above the plot. The parameters for the dispersed phase are $\left|L^{\prime} a\right|=1.24|L a|$ and $K^{\prime} a=1.05 K a$.

the scattering calculation is in addition to the nonscattering contribution. Figures 1 and 2 show the velocity and attenuation per wavelength $(\alpha \lambda)$ as a function of frequency in the form of the parameters $K a$ and $L a$. The ultrasound properties have been calculated by four different methods, including ECAH and the general theory results presented here.

It was found that the LFPST theory (Harlen et al. [4]) for low frequencies required orders up to $m=8$ in order to obtain even the first part of the change in velocity and attenuation as the frequency increases. The theory is very much confined to the lowest frequency range. Similarly the geometric theory (Harlen et al. [5]) (which here was calculated only to second order in $m$ and $n$ ) is valid only for high frequencies within the long wavelength region and deviates from the ECAH values as the frequency decreases.

The general theory presented in the current work is valid over the entire frequency range within the long wavelength region. The results for velocity and attenuation match closely those determined using the ECAH method. Visco-inertial scattering, 


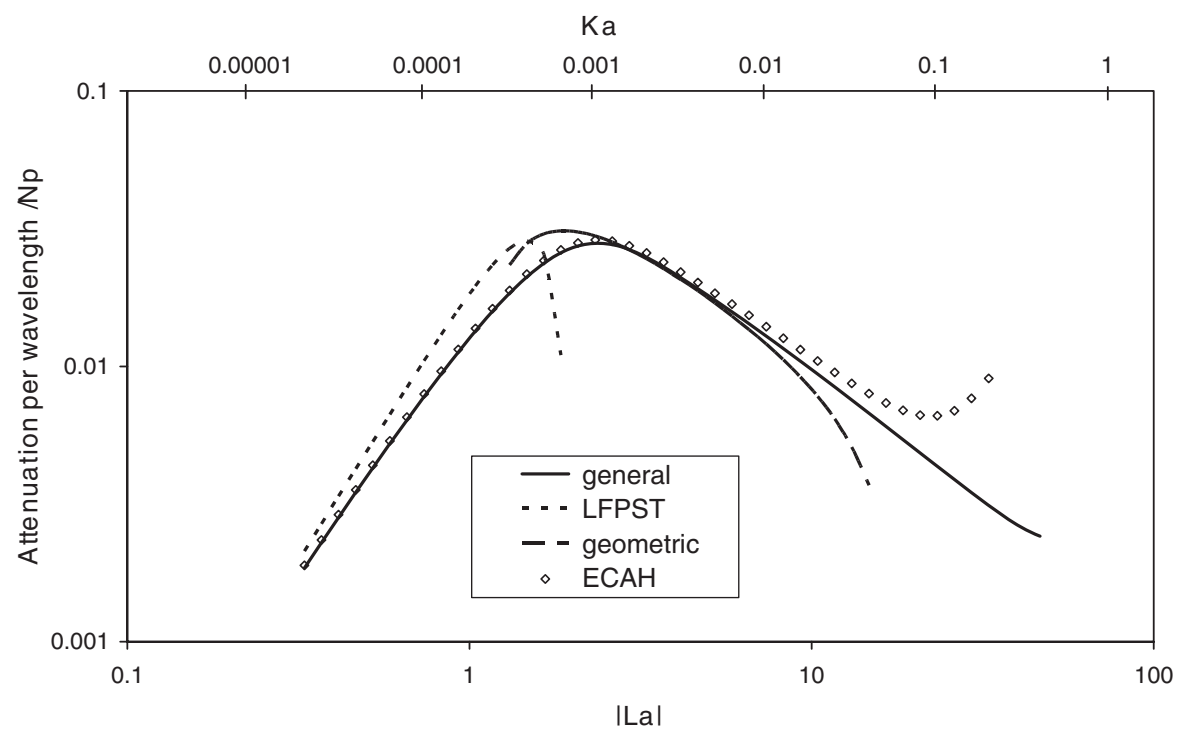

FIG. 2. Attenuation per wavelength as a function of the parameter $\mid$ La $\mid$ for $20 \%$ sunflower oil in water at $30^{\circ} \mathrm{C}$. Four different calculation methods are shown: the "general" method presented in this paper, the LFPST method (Harlen et al. [4]), the geometric theory method (Harlen et al. [5]), and the ECAH method (Epstein and Carhart [2]). Corresponding values of Ka are shown above the plot. The parameters for the dispersed phase are $\left|L^{\prime} a\right|=1.24|L a|$ and $K^{\prime} a=1.05 K a$.

which relates to a difference in density between the two components and their viscosity, is not included in the general theory but is included in ECAH. The densities of the two components are similar, but the additional scattering accounts for the difference between the general theory and ECAH results. Terms for $m \leq 4$ were included for the general theory, as higher orders did not significantly change the result. At higher frequencies, the long wavelength criterion $(K a \ll 1)$ is no longer valid, and more and more terms are needed to obtain an accurate result. Figure 3 shows the contribution of including these terms. The second order solution is very accurate for frequencies below the point $K a=0.007$. The fourth order solution gives a more accurate result over a much wider frequency range.

7. Conclusions. A method has been presented for the solution of the ultrasound scattering problem in the long wavelength region. The work builds on previously published studies which covered only part of the frequency range, when the thermal wavelength is either much smaller or much larger than the particle size. The technique consists of expressing the scattered fields as perturbation series in the parameter $K a$, which is always small in the long wavelength region, and explicitly removing the radiating field factor $e^{i K r}$. A result has been obtained which covers the complete long wavelength region. The calculation is much more straightforward than the widely used ECAH method, which relies on spherical harmonic expansions and suffers from numerical instability. A simplified analytical version of the result has been produced which enables calculation in an ordinary spreadsheet.

8. Appendix. The thermal wave solutions use expansions of the spherical Bessel functions which are not generally found in mathematical texts. The coefficients can be calculated in the order $n$ by the formula below. 


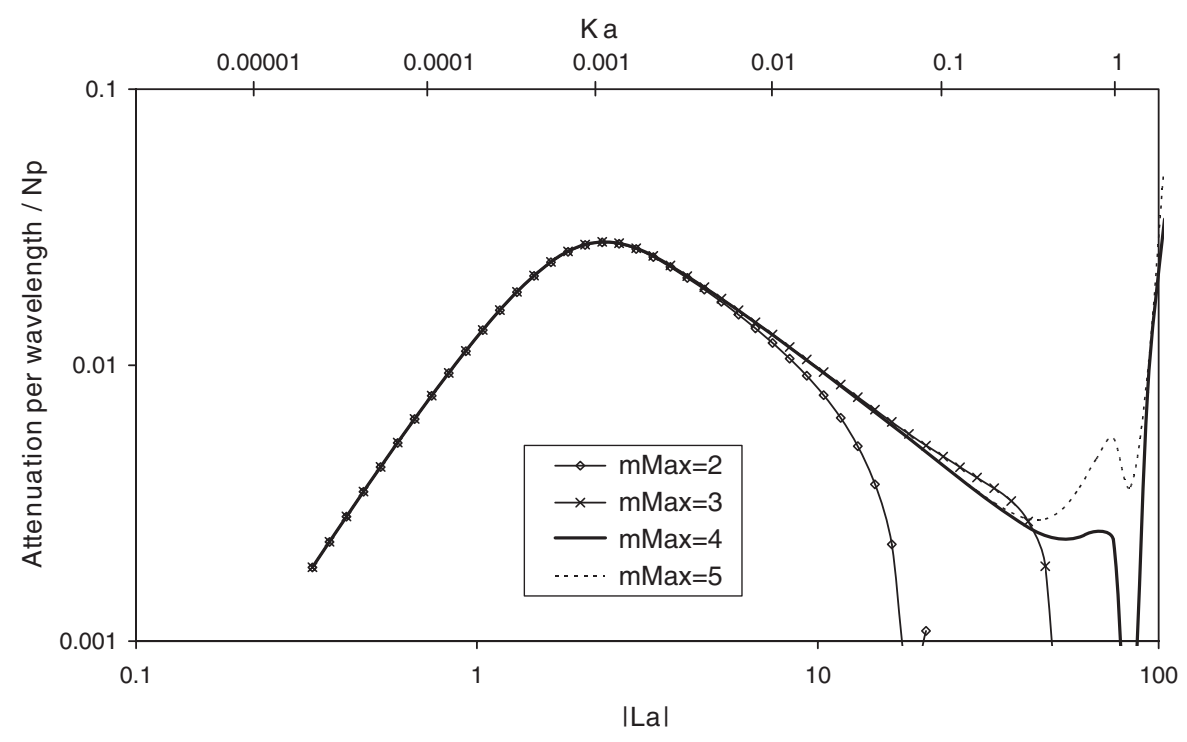

FIG. 3. General theory results for $20 \%$ sunflower oil in water at $30^{\circ} \mathrm{C}$ showing the contributions of higher order terms in the series expansion, especially at larger values of $K a$. The parameters for the dispersed phase are $\left|L^{\prime} a\right|=1.24|L a|$ and $K^{\prime} a=1.05 K a$.

For the spherical Hankel function,

$$
\begin{aligned}
& h_{01}=-i \text { for } n=0 \\
& \text { for } n>0 \quad h_{n, j}=\left\{\begin{array}{l}
-i h_{n-1, j} \text { for } j=1, \\
-i h_{n-1, j}+(n+j-2) h_{n-1, j-1} \text { for } 1<j<n+1, \\
(2 n-1) h_{n-1, j-1} \text { for } j=n+1 .
\end{array}\right.
\end{aligned}
$$

Similarly for the spherical Bessel function, which was defined in two parts, an outgoing and an ingoing traveling wave:

$$
\begin{aligned}
& j_{0,1+}=1 / 2 \text { i for } n=0, \\
& \text { for } n>0 \quad j_{n, j+}=\left\{\begin{array}{l}
-i j_{n-1, j+} \text { for } j=1, \\
-i j_{n-1, j+}+(n+j-2) j_{n-1, j-1+} \text { for } 1<j<n+1, \\
(2 n-1) j_{n-1, j-1+} \text { for } j=n+1,
\end{array}\right. \\
& j_{0,1-}=1 / 2 \text { i for } n=0, \\
& \text { for } n>0 \quad j_{n, j-}=\left\{\begin{array}{l}
i j_{n-1, j-} \text { for } j=1, \\
i j_{n-1, j-}+(n+j-2) j_{n-1, j-1-} \text { for } 1<j<n+1, \\
(2 n-1) j_{n-1, j-1-} \text { for } j=n+1 .
\end{array}\right.
\end{aligned}
$$

\section{REFERENCES}

[1] J. W. Strutt (Baron Rayleigh), The Theory of Sound, 2nd ed., Macmillan, London, 1896.

[2] P. S. EPSTEIN AND R. R. CARHART, The absorption of sound in suspensions and emulsions. I. Water fog in air, J. Acoust. Soc. Amer., 25 (1953), pp. 553-565. 
[3] J. R. Allegra and S. A. Hawley, Attenuation of sound in suspensions and emulsions: Theory and experiments, J. Acoust. Soc. Amer., 51 (1972), pp. 1545-1564.

[4] O. G. Harlen, M. J. Holmes, M. J. W. Povey, Y. Qiu, and B. D. Sleeman, A low frequency potential scattering description of acoustic propagation in dispersions, SIAM J. Appl. Math., 61 (2001), pp. 1906-1931.

[5] O. G. Harlen, M. J. Holmes, M. J. W. Povey, and B. D. Sleeman, Acoustic propagation in dispersions and the geometric theory of diffraction, SIAM J. Appl. Math., 63 (2003), pp. 834-849.

[6] D. Colton And R. Kress, Inverse Acoustic and Electromagnetic Scattering Theory, 2nd ed., Springer, Berlin, 1998.

[7] G. Arfken, Mathematical Methods for Physicists, 3rd ed., Academic Press, Orlando, FL, 1985.

[8] P. Lloyd AND M. V. BerRy, Wave propagation through an assembly of spheres. IV. Relations between different multiple scattering theories, Proc. Phys. Soc., 91 (1967), pp. 678-688.

[9] P. C. Waterman and R. Truell, Multiple scattering of waves, J. Math. Phys., 2 (1961), pp. 512-537.

[10] J. G. Fikioris And P. C. Waterman, Multiple scattering of waves. II. "Hole corrections" in the scalar case, J. Math. Phys., 5 (1964), pp. 1413-1420. 